\title{
Chaotic, memory, and cooling rate effects in spin glasses: Evaluation of the Edwards-Anderson model
}

\author{
Marco Picco* \\ LPTHE, Université Pierre et Marie Curie, Paris VI, Université Denis Diderot, Paris VII, Boite 126, Tour 16, $1^{\text {er }}$ étage, 4 place Jussieu, \\ F-75252 Paris Cedex 05, France \\ Federico Ricci-Tersenghi ${ }^{\dagger}$ \\ Abdus Salam International Center for Theoretical Physics, Condensed Matter Group, Strada Costiera 11, P.O. Box 586, \\ I-34100 Trieste, Italy \\ Felix Ritort ${ }^{*}$ \\ Department of Physics, Faculty of Physics, University of Barcelona, Diagonal 647, 08028 Barcelona, Spain
}

(Received 5 June 2000; revised manuscript received 8 November 2000; published 5 April 2001)

\begin{abstract}
We investigate chaotic, memory, and cooling rate effects in the three-dimensional Edwards-Anderson model by doing thermoremanent (TRM) and ac susceptibility numerical experiments and making a detailed comparison with laboratory experiments on spin glasses. In contrast to the experiments, the Edwards-Anderson model does not show any trace of reinitialization processes in temperature change experiments (TRM or ac). A detailed comparison with ac relaxation experiments in the presence of dc magnetic field or coupling distribution perturbations reveals that the absence of chaotic effects in the Edwards-Anderson model is a consequence of the presence of strong cooling rate effects. We discuss possible solutions to this discrepancy, in particular the smallness of the time scales reached in numerical experiments, but we also question the validity of the Edwards-Anderson model to reproduce the experimental results.
\end{abstract}

DOI: 10.1103/PhysRevB.63.174412

PACS number(s): 75.10.Nr, 05.50.+q, 75.40.Gb, 75.40.Mg

\section{INTRODUCTION}

One of the most characteristic effects in disordered or glassy systems in their non-stationary regime is the presence of aging. The response of the system stiffens with age showing that it depends on all the previous history through, e.g., the waiting time. ${ }^{1,2}$ Experimentally, this phenomenon is well documented through magnetization relaxation experiments and ac susceptibility measurements. ${ }^{3-5}$ Despite the different experimental procedures needed for both type of measurements, magnetization relaxation and ac susceptibility give similar information regarding the aging behavior and its waiting time dependence.

On top of all these nonequilibrium phenomenology, recent dynamical experiments in spin glasses show very peculiar chaotic (also called rejuvenation), memory as well as cooling rate effects. ${ }^{6-8}$ These effects are thought to be the signature of the spin glass state being much different to those found in usual ferromagnets or other disordered systems. ${ }^{9}$ The most unusual experimental result in spin glasses is the absence of cooling rate effects. The approach to equilibrium at a given temperature after cooling from high temperatures is not influenced by the whole cooling history at higher temperatures but only by the time spent at the last temperature in the thermal history. Experimentally, rejuvenation or chaotic effects in spin glasses are measured in a clear way by doing ac measurements. An alternating magnetic field of frequency $\omega=1 / P$ where $P$ is the period applied to the sample and both components of the ac susceptibility (the in-phase $\chi^{\prime}$ and the out-of-phase $\chi^{\prime \prime}$ ) are measured.

Although the major part of these measurements have been done on insulating spin glasses they are common also to metallic spin glasses leading to the question whether these effects are also present in the most well known theoretical models. Despite of the large amount of theoretical work devoted to aging effects in glasses and spin glasses there is still no convincing and final explanation for the origin of these peculiar chaotic and memory effects. The comprehension of these effects will certainly provide a clue to the understanding of the nature of the glassy state.

Because memory and chaotic (or rejuvenation) effects are intrinsic to spin glasses (metallic and insulating) it is important to understand whether models for spin glasses are able to reproduce the experimental results. It is widely accepted that the Edwards-Anderson model contains the main features observed in real spin glasses. The purpose of this paper is to present a detailed and critical study of these phenomena in the Edwards-Anderson model in three dimensions. This is not a simple matter to address. Despite of the large amount of numerical studies on equilibrium and nonequilibrium phenomena there is no clear evidence that the EdwardsAnderson model reproduces the main results found in experiments. Note that even the question whether there is or not phase transition in the 3D Edwards-Anderson model is still not fully settled. ${ }^{10,11}$

The purpose of this paper is to present a numerical investigation, fully experimentally oriented, of the nonequilibrium behavior of the three dimensional Ising spin glass with special emphasis on recent experiments where memory and chaos effects where found. This question is of the utmost importance concerning modeling. If some experimentally observed results are missing in any theory then we must understand why. There have been several investigations in the literature devoted to this subject, but still a clear answer is 
missing. ${ }^{12-14}$ Here we will provide a complementary investigation to results already published, emphasizing the experimental results and comparing different types of experiments. In particular, our main effort will be devoted to investigate thermoremanent and ac numerical experiments. Although thermoremanent studies have been largely considered in the past there are very few numerical investigations devoted to the ac topic. ${ }^{15}$

The paper is divided as follows. In Sec. II we introduce the model as well as the dynamical procedure. Section III discusses the two type of measurements we have done: magnetization relaxation and ac numerical experiments. Sections $\mathrm{IV}$ and $\mathrm{V}$ present a detailed investigation of memory and chaotic effects with thermoremanent and ac experiments respectively. Finally we present a discussion of the results.

\section{THE EDWARDS-ANDERSON MODEL AND SOME DETAILS OF THE SIMULATION}

The Edwards-Anderson mode ${ }^{16}$ was proposed in the early 1970s as the simplest model which contains the main ingredients relevant to explain the spin-glass phenomenology. In particular, it displays a phase transition characterized by the onset of freezing in spin-spin correlations and a divergent nonlinear susceptibility. ${ }^{17}$ The model is defined by the following Hamiltonian:

$$
\mathcal{H}=-\sum_{(i, j)} J_{i j} \sigma_{i} \sigma_{j}-h \sum_{i=1}^{V} \sigma_{i},
$$

where the indices $i, j$ run from 1 to $\mathrm{V}$, the $\sigma_{i}$ are Ising spins and the pairs $(i, j)$ identify nearest neighbors in a finite dimensional lattice. The exchange couplings $J_{i j}$ are taken from a random distribution. To avoid degeneracy of the ground state the simplest choice is a Gaussian distribution with zero average and finite variance,

$$
\mathcal{P}(J)=\left(\frac{1}{2 \pi \Delta^{2}}\right)^{1 / 2} \exp \left(-\frac{J^{2}}{2 \Delta^{2}}\right) .
$$

The model is defined in any number of finite dimensions although our main concern here is the three-dimensional case where there is a spin glass transition at finite temperature $T_{c} \simeq 0.95 \Delta .{ }^{10}$ Hereafter, unless differently specified, we will consider $\Delta=1$ without loss of generality.

Monte Carlo simulations of Eq. (1) use random updating of the spins with the Metropolis algorithm. A spin is randomly chosen and its value changed with the proper probability. Dynamical experiments use very large lattices (typical sizes are in the range $L=20-100)$ with negligible finitesize effects for the largest sizes $(L=64$ for magnetization relaxation experiments and $L=100$ for ac experiments). Here we present two classes of different but related experiments. Magnetization and correlation relaxation simulations have run on a special purpose machine APE (Ref. 19) for sizes $64^{3}$ and averaging over 10 or 100 samples. ac experiments were run for a single sample on a Linux cluster of PCs for sizes $L=64$ and 100 .
Before presenting the results it is convenient to discuss the fidelity of the EA model to real spin glasses. Clearly, the EA model is an idealization of the real microscopic interaction found in spin glasses. ${ }^{17}$ Spin glasses are commonly distinguished into two large classes: metallic and insulating. Metallic spin glasses are diluted magnets where a metallic host matrix is doped with some ferromagnetic impurities (for instance $\mathrm{AgMn}, \mathrm{AuFe}, \mathrm{CuMn}$ ). In these systems spin interactions are due to indirect exchange and mediated through conduction electrons (the RKKY interaction). Metallic spin glasses are then diluted magnets where site disorder induces frustrated short-ranged interactions (decaying like $1 / r^{3}$ with $r$ being the distance between impurities). Insulating spin glasses are much different. In this case, exchange interactions are usually antiferromagnetic between neighbor spins but dilution and defects lead to a strong frustration. Apart from the different microscopic origin of the frustrating interaction, spins are really Heisenberg-like and the Ising behavior arises from the uniaxial anisotropy present in these type of systems. Because anisotropy is usually strong and the local rotational symmetry of Heisenberg spins is broken, a treatment taking pure Ising spins turns out to be a good approximation. ${ }^{18}$ Having in mind these limitations, the Hamiltonian (1) is the simplest model which contains disorder and frustration, the two ingredients commonly found in real spin glasses.

\section{MAGNETIZATION RELAXATION AND AC EXPERIMENTS}

There are two alternative but equivalent ways to experimentally investigate nonequilibrium phenomena in spin glasses: magnetization relaxation experiments and ac measurements. A very complete description of these methods can be found in Ref. 3. Here we only remind the main results.

\section{Magnetization relaxation (TRM) experiments}

Relaxation measurements are done applying a uniform magnetic field and measuring the decay of the thermoremanent magnetization (hereafter referred to as TRM), equivalently, the growth of the zero-field cooled magnetization. The typical experiment consists in the following. A sample is fastly quenched below the spin glass transition temperature for a time $t_{w}$ (i.e., the waiting time). Then a uniform small magnetic field $h$ is applied and the growth of the magnetization measured,

$$
\chi\left(t_{w}, t_{w}+t\right)=\frac{1}{V h} \sum_{i=1}^{V} \sigma_{i}\left(t_{w}+t\right) .
$$

In the linear response regime Eq. (3) can be written as

$$
\chi\left(t_{w}, t_{w}+t\right)=\int_{t_{w}}^{t_{w}+t} R\left(t_{w}+t, s\right) d s
$$

where $R(t, s)$ is the response function which gives the change of the magnetization $\delta M$ at time $t$ when a pulse of the magnetic field $\delta h$ is applied at previous time $s$. In spin 
glasses aging manifests by the fact that $\chi\left(t_{w}, t_{w}+t\right)$ shows a strong dependence on the value of $t_{w}$. In general, one finds the following decomposition:

$$
\chi\left(t_{w}, t_{w}+t\right)=\chi_{s t}(t)+\chi_{a g}\left(t_{w}, t_{w}+t\right),
$$

where $\chi_{s t}$ and $\chi_{a g}$ are respectively the stationary and aging parts. Experimentally the aging part approximately scales with the waiting time $t_{w}$ in the following way:

$$
\chi_{a g}\left(t_{w}, t_{w}+t\right)=f\left(t / t_{w}\right)
$$

although systematic deviations from this scaling behavior have been observed. This point will be discussed later on.

Related to magnetization another quantity of interest which can be numerically investigated in simulation are twotime correlations. These quantities are difficult to experimentally measure in spin glasses but very easy to compute in simulations. They are defined by

$$
C\left(t_{w}, t_{w}+t\right)=\frac{1}{V} \sum_{i=1}^{V} \sigma_{i}\left(t_{w}\right) \sigma_{i}\left(t_{w}+t\right) .
$$

Again, in the nonequilibrium regime Eq. (7) can be decomposed in two pieces, a stationary part plus an aging part

$$
C\left(t_{w}, t_{w}+t\right)=C_{s t}(t)+C_{a g}\left(t_{w}, t_{w}+t\right)
$$

Similar to the magnetization, the aging part of the correlation is approximately described by the following scaling behavior:

$$
C_{a g}\left(t_{w}, t_{w}+t\right)=g\left(t / t_{w}\right),
$$

again with systematic (but small) deviations respect to it. The stationary part of the correlation and magnetization are related through the fluctuation-dissipation theorem (FDT)

$$
\chi_{s t}(t)=\frac{1-C_{s t}(t)}{T} .
$$

Although in mean-field spin glasses a more general relation seems to be valid. ${ }^{5}$ It links response and correlation functions also in the off-equilibrium regime through

$$
X[C]=-\left.T \frac{\partial \chi(s, t)}{\partial C(s, t)}\right|_{C(s, t)=C\left(t_{w}, t_{w}+t\right)},
$$

where the fluctuation-dissipation ratio $X$ depends only on the correlation function in the large times limit $\left(t, t_{w} \rightarrow \infty\right)$. In the quasiequilibrium regime $\left(t<t_{w}\right)$ we have that $X=1$ and we recover the usual FDT. In the aging regime $\left(t>t_{w}\right)$ the ratio is smaller than one $X<1$ and it can be interpreted as a larger effective temperature $T_{\text {eff }} \sim T / X$. In finite-dimensional spin glasses the validity of Eq. (11) has been numerically checked $^{20,21}$ and it has been related to the equilibrium distribution of overlaps. ${ }^{21}$

\section{ac measurements}

In these experiments an oscillating magnetic field $h(t)$ $=h_{0} \cos (2 \pi \omega t)$ of frequency $\omega=1 / P$, where $P$ is the period, is applied to the system and the magnetization measured as a function of time

$$
M(t)=M_{0} \cos (2 \pi \omega t+\phi),
$$

where $M_{0}$ is the intensity of the magnetization and $\phi$ is the dephasing between the magnetization and the field. The origin of the dephasing is dissipation in the system which prevents the magnetization to follow the oscillations of the magnetic field. From the magnetization one can obtain the inphase and out-of-phase susceptibilities defined as

$$
\begin{gathered}
\chi^{\prime}=\frac{M_{0} \cos (\phi)}{h_{0}}=\frac{2 \int_{0}^{P} M(t) \cos (2 \pi \omega t) d t}{h_{0}}, \\
\chi^{\prime \prime}=\frac{M_{0} \sin (\phi)}{h_{0}}=\frac{2 \int_{0}^{P} M(t) \sin (2 \pi \omega t) d t}{h_{0}} .
\end{gathered}
$$

The dephasing $\phi$ measures the rate of dissipation in the system and is given by

$$
\tan (\phi)=\frac{\chi^{\prime \prime}}{\chi^{\prime}}
$$

In numerical simulations the in-phase and out-of-phase susceptibilities are computed by averaging the right-hand side in Eqs. (13) and (14) over several periods $P=1 / \omega$. This means a very large measurement time for low frequencies for both experiments and simulations. In the nonequilibrium regime the ac susceptibility ${ }^{22}$ depends on both the waiting time and the frequency. On general grounds one expects that

$$
\chi(\omega, t)=\chi_{s t}(\omega)+\chi_{a g}(\omega, t),
$$

where the aging part of the ac susceptibility approximately satisfies a scaling behavior

$$
\chi_{a g}(\omega, t) \sim h(\omega t)
$$

Both types of measurements give equivalent information about the relaxation dynamics but in different time sectors. As discussed in Ref. 3, TRM experiments give information on time scales ranging between the two limits $t \ll t_{w}$ and $t$ $\gg t_{w}$. For ac experiments the frequency $\omega$ corresponds to the inverse of the observation time $t_{o b s}$ (note that in TRM experiments after switching the field we have that $t_{o b s}=t$ ) while the age $t_{a}$ corresponds to the total elapsed time $t$ $+t_{w}$. In ac experiments in order to get reliable results on $\chi^{\prime}$ and $\chi^{\prime \prime}$ one needs to average over several periods of the field, while keeping the age of the system more or less unaltered (otherwise the two limiting regimes would mixed and the results would be unclear). This is possible only if $\omega t_{a} \gg 1$, which imply $t \ll t_{w}$. Consequently, in ac measurements one is able only to explore the beginning of the aging regime, 
also called quasistationary regime ${ }^{23}$ which represents a smaller time window than in TRM experiments.

\section{MEMORY AND CHAOS IN CORRELATION AND RESPONSE FUNCTIONS}

In this section we perform a study of memory and chaos effect in spin glasses measuring correlation and response functions. We always take the measurements from 10 samples of a $64^{3}$ system (unless differently specified). We closely follow the experimental procedure on what concerns temperature changes and we keep the ratio between time scales entering in the simulation similar to the experimental ones (with the same limitations of sizes, magnetic fields and absolute time scales as already discussed).

\section{A. "Cooling and stop" experiment}

The experiment is performed in the following way (for more details the reader is address to the original paper ${ }^{6}$ ). Starting from the high temperature phase, a spin glass sample is cooled at a fixed cooling rate into the glassy phase. When a temperature $T^{*} \simeq 0.8 T_{c}$ is reached, the cooling process is stopped and the sample is let to relax for a long time. This relaxation produces a decrease in the susceptibility (in both $\chi^{\prime}$ and $\chi^{\prime \prime}$ ) with respect to the reference curve (obtained with the same constant cooling rate and without any stop). After that long time the cooling process is continued down to a low temperature and finally the sample is heated back again at a constant heating rate (equal to the cooling one) and without any stop.

There are two relevant results in this experiment. First, as soon as the cooling process is started again after the stop, the susceptibility merges rapidly with the reference curve, quickly "forgetting" the thermalization work done near the temperature $T^{*}$ (chaos effect). Second, when the sample is heated back at a fixed heating rate and without any stop, the susceptibility closely follows the cooling curve and it goes through the dip at $T^{*}$ (memory effect).

In our simulations we do an analogous experiment, measuring correlation functions instead of susceptibilities. We divide the time of the experiment in intervals of duration $P$ (here two values $P=10^{3}, 10^{4}$ will be considered) and we measure the correlation function between the first and the last times in the interval, $C(0, P)$. This correlation is strictly related to the in-phase susceptibility, $\chi^{\prime}=(1-C) / T$, measured with an external magnetic field of frequency $\omega \propto 1 / P$. In order to confirm this relation we show in the inset of Fig. 1 the susceptibilities measured in two reference experiments without any stop and with cooling and heating rates such that we perform $P$ MCS at every temperature and then we change the temperature by $\Delta T=0.02$. The curves resemble very much the experimental ones. Compared to the ac measurements, simulations with the correlations have the advantage that one can reach higher values of $P$, i.e., lower frequencies. Note that, because of the precision required in this experiment, all the susceptibilities have been averaged over 100 samples of size $64^{3}$. In the inset of Fig. 1 some effects due to the finite cooling rates can also be appreciated. In particular,

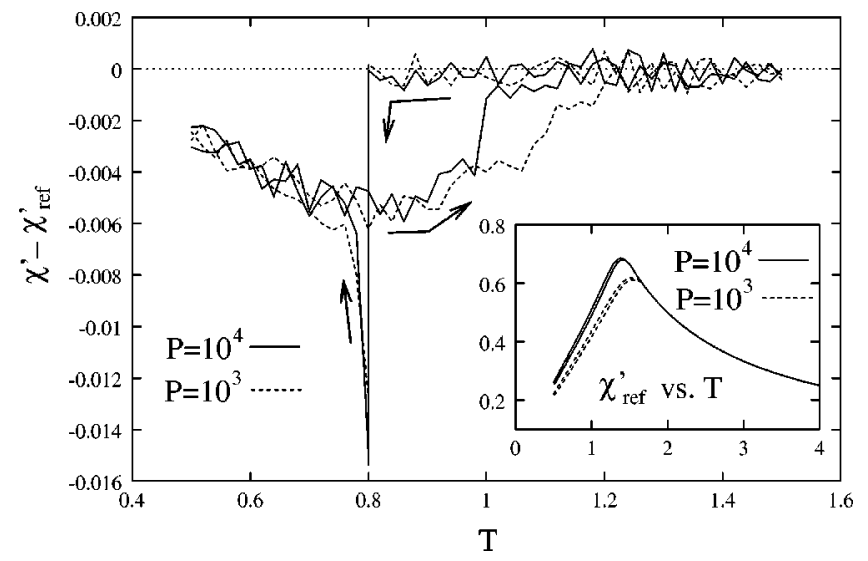

FIG. 1. The "cooling and stop" experiment in the EA model gives no evidence for such strong chaos and memory effects experimentally found on real samples. Here we use 100 samples of a $64^{3}$ system, cooling rates inversely proportional to $P$ and probing time scales $\mathcal{O}(P)$. In the inset we show the reference curves measured without any stop during the cooling.

one can see that the curves measured during the cooling processes stay a little bit above the corresponding curves measured during the heating process. This means that the system "accumulates" part of the relaxation work done in the lowtemperature phase. The cooling and heating curves merge together only when the system comes back to the hightemperature phase. This phenomenon is present also in experimental spin glasses, even if with a much smaller intensity. ${ }^{24}$

The interesting information can be obtained once we perform a long stop in the spin-glass phase. Here we stop the cooling at a temperature $T^{*}=0.8$ for $t_{w}=100 \cdot P$ MCS (i.e., 100 intervals) and measure $\chi^{\prime}$ from $C\left(t_{w}, t_{w}+P\right)$. The system relaxes and $\chi^{\prime}-\chi_{\text {ref }}^{\prime}$ becomes negative. However, when we start again cooling the system, the susceptibility does not recover completely the reference curve and it always remain with smaller values. Note that at the experimental level ${ }^{6,8}$ the susceptibility recovers the reference value very rapidly (around $T \simeq 0.65-0.7$ on the scale of Fig. 1) and that the apparent rapid increase just below $T^{*}$ in Fig. 1 is due to the very zoomed $y$ axis and it has in fact a slope of order one. Moreover the curve followed by the data for $T<T^{*}$ does not seem to depend on $P$ and if we consider the relative difference, $\left(\chi^{\prime}-\chi_{\text {ref }}^{\prime}\right) / \chi_{\text {ref }}^{\prime}$, we would obtain that the convergence towards 0 is still slower, due to the fact that both susceptibilities are decreasing with $T$.

During the heating process the system stays on the same cooling curve and it does not show any strange effect near $T^{*}$. For $T>T^{*}$ it finally recovers the reference curve and here is where we observe the largest dependence on $P$. The temperature where $\chi^{\prime}$ becomes comparable with $\chi_{\text {ref }}^{\prime}$ strongly decreases with increasing $P$. Nevertheless for the times we have access to $\left(P=10^{3}, 10^{4}\right)$ this temperature is larger than the critical one $T_{c} \simeq 0.95$ and in the limit of large times, $P \rightarrow \infty$, it can converge to both $T_{c}$ or $T^{*}$.

In conclusion we can assert that the three-dimensional Edwards-Anderson model does not show, on the time scales we have access to, the strong memory and chaos effects real spin glasses show. 
The same numerical experiment we have presented in this section have been recently done also by Komori et al. (see Fig. 7 in Ref. 14). In that figure it is shown that some kind of memory and chaotic effects are found when measuring the out-of-phase component of the AC susceptibility. But that figure and all the subsequent authors discussion based on it are inconclusive for the following reasons. They use a magnetic field that oscillates too fast $(P=160)$. As a consequence the effective critical temperature is very high, $T_{c}^{\mathrm{eff}}$ $>2.5 \simeq 3 T_{c}$. Moreover they use a cooling rate which is 10 times higher compared to experimental protocols. Each point in their figure is a measure over a single cycle of the field and a single dynamical history averaged over many different samples. ${ }^{25}$ Finally they claim to see in their Fig. 7 a merge of the susceptibility data to the reference curve, which is far from evident without any zoom of the interesting region. Note that both susceptibilities ( $\chi$ and $\chi_{\text {ref }}$ ) goes to zero when $T \rightarrow 0$ and then we also expect $\chi-\chi_{\text {ref }}$ to become zero. One should check that the relative difference is going to zero faster in order to claim for the presence of chaotic effects. In our study we had to increase the precision of more than 2 orders of magnitude (note the $y$-axis scale in Fig. 1) in order to discern the effect. The deceiving result is that, if the experiment corresponding to Fig. 7 of Ref. 14 is done with slower cooling rates (unfortunately, such results were not shown in Ref. 14), one does not probably observe any trace of rejuvenation or memory due to the strong cooling rate effects. This is definitely different from what experiments show.

\section{B. Temperature cycling experiments}

In another set of very interesting experiments the temperature is changed according to the following scheduling: $t_{w 1}$ seconds at $T_{1}$, then $t_{w 2}$ at $T_{2}$ and finally $t_{w 3}$ at $T_{1}$ again. After that the TRM decay is measured. Depending on the sign of $\Delta T=T_{2}-T_{1}$ the system responds in different ways. For $\Delta T<0$ an effective waiting time, $t_{w}^{\text {eff }}$, in the TRM decay can be defined and it is a monotonic function of $\Delta T$ such that $t_{w}^{\text {eff }}=t_{w 1}+t_{w 2}+t_{w 3}$ for $\Delta T=0$ and $t_{w}^{\text {eff }}=t_{w 1}+t_{w 3}$ for $|\Delta T|$ large. For $\Delta T>0$ the TRM decay follow a more complicated law and it cannot be described just by an effective waiting time. Nevertheless is always possible to define a correlation time, which turns out to be a monotonic function of $\Delta T$, taking the same value as before for $\Delta T=0$, but converging to $t_{w}^{\text {eff }}=t_{w 3}$ for large $|\Delta T|$.

In numerical simulations the effective waiting time (or correlation time) can be estimated from the decay of the correlation function. In the inset of Fig. 2 we show the correlation measured in a cycling temperature experiment (with $T_{1}=0.7$ and $\left.T_{2}=0.9\right)$ where the relative times are similar to those used by experimentalists $\left(t_{w 1}=10^{4}, t_{w 2}=10^{2}\right.$ and $t_{w 3}=10^{2}$ ). The reference curve with $\Delta T=0$ will always refer to data measured at fixed temperature $T_{1}$ and with a waiting time $t_{w}=t_{w 1}+t_{w 3}$. As it is clear from the data the temperature cycle does not affect at all the decay of the correlation function and the system does not seem to be reinitialized. This effect is still more drastic if we use a different scheduling in order to amplify it. In the main body of

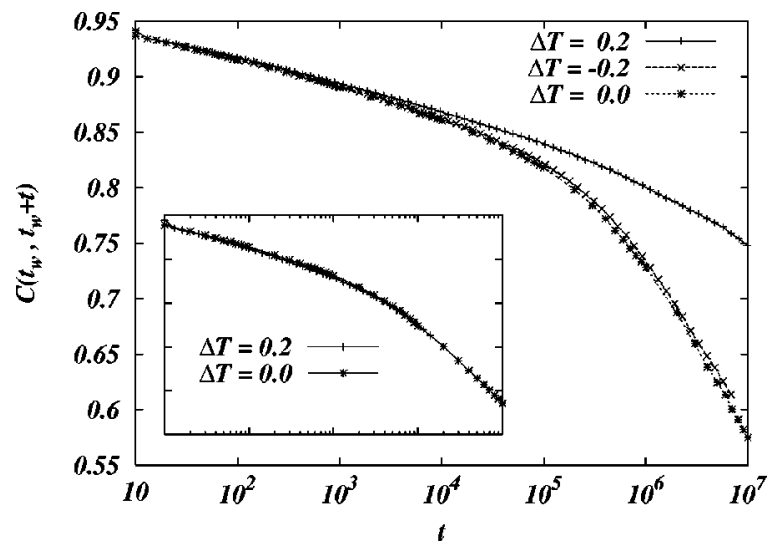

FIG. 2. Correlation relaxation in temperature cycling experiments. In the inset we have used temperatures and time scale ratios similar to the experimental ones (see text for details) and we do not see any difference with respect to the reference curve. In the main part we show the results for a large perturbation both in temperature, $\Delta T= \pm 0.2$, and in times, $t_{w 2}=t_{w 1}+t_{w 3}=10^{6}$. It is clear that for both positive and negative cycles the system is more thermalized with respect to the reference system where $t_{w}=10^{6}$.

Fig. 2 we report the correlations measured with $t_{w 1}=5$ $\times 10^{5}, t_{w 2}=10^{6}$ and $t_{w 3}=5 \times 10^{5}$, with $T_{1}=0.5$ and $T_{2}$ $=0.7$ or 0.3 . From the data it is clear that the effective waiting time is increased in both cases in contrast to what it is observed in experiments, being the time spent at the higher temperature $T_{2}=0.7$ much more effective in terms of the thermalization process.

We expect the effective waiting time to be more or less related to the size of thermalized regions in the system. Once we have characterized how this size changes under a temperature cycle, we can also study how the internal structure of these thermalized regions is modified by the cycle. In order to do this we exploit the generalization of the fluctuation-dissipation relation to the off-equilibrium regime discussed in Sec. III. In Fig. 3 we show the off-equilibrium susceptibility versus the correlation for the same experiments reported in Fig. 2 and described in the previous paragraph. The temperature cycle, even when it is very long, does not

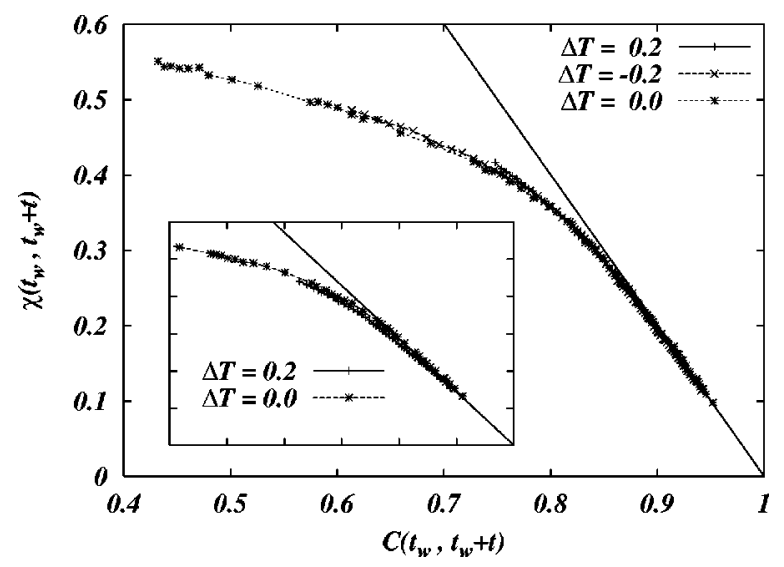

FIG. 3. Fluctuation-dissipation ratio measured in the experiments of Fig. 2. The (effective) temperature seems to be unchanged by the temperature cycle. 


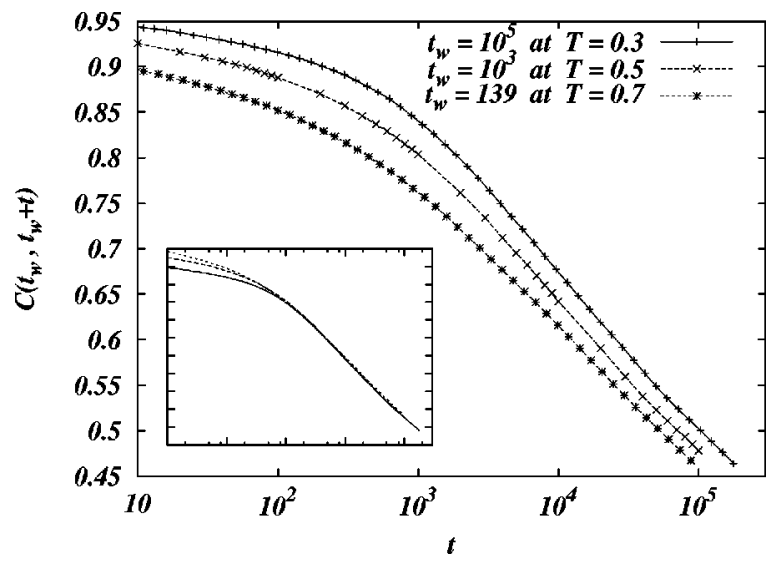

FIG. 4. The decays of the correlation function at temperature $T=0.5$, after the thermalizations shown in the legend, have the same effective waiting time (as can be seen in the inset where we have rescaled the curves by means of simple multiplicative factors). The temperatures and the waiting times (see legend) have been chosen such that the size of thermalized regions, $\xi(t, T) \propto t^{a T}$ [with $a=0.161$ (Ref. 27) and a prefactor of order 1], is comparable.

seem to affect the response of the system. In the quasiequilibrium regime the slope of the curve $\chi$ versus $C$ gives the temperature in the thermalized regions. This temperature does not seem to change even if the system spends a lot of time in a different temperature $T_{2}$. When it comes back to $T_{1}$ it rapidly seems to recover the configuration corresponding to temperature $T_{1}$.

\section{Temperature shift experiments}

In order to better understand how the time the system spends at a temperature $T_{1}$ can influence the thermalization process at a different temperature $T_{2}$ we have performed a series of temperature shift experiments. Here the scheduling is the following. After $t_{w 1}$ MCS at temperature $T_{1}$ we set $T=T_{2}$ and we immediately start measuring the correlation and the response to a small external field. In the present study $T_{2}=0.5$ and $T_{1}=0.7,0.5,0.3$, the second case being considered as a reference curve. Moreover the waiting times, $t_{w 1}=139,10^{3}, 10^{5}$, have been chosen such that $t_{w 1}^{T_{1}}$ is constant. It is known that in the Edwards-Anderson model the dynamical correlation length grows as a power law of time, $\xi \propto t^{1 / z(T)}$, where the dynamical exponent $z(T)$ is inversely proportional to the temperature. ${ }^{26,27}$ So our choice for the waiting times would correspond to thermalized regions of similar sizes.

In Fig. 4 we see that the effective waiting time generated by the three different scheduling is very similar. The three curves can be perfectly collapse in the aging regime by simply multiplying them by a constant (see inset in Fig. 4). This means that the effective waiting time is essentially given by the size of the thermalized regions, which has been chosen to be equal in the three experiments. The difference between the three curves in Fig. 4 comes from the quasiequilibrium part $C_{s t}(t)$, which decays in a different way.

The following natural question concerns the configuration of the system up to length scales of the order of $\xi(t)$.

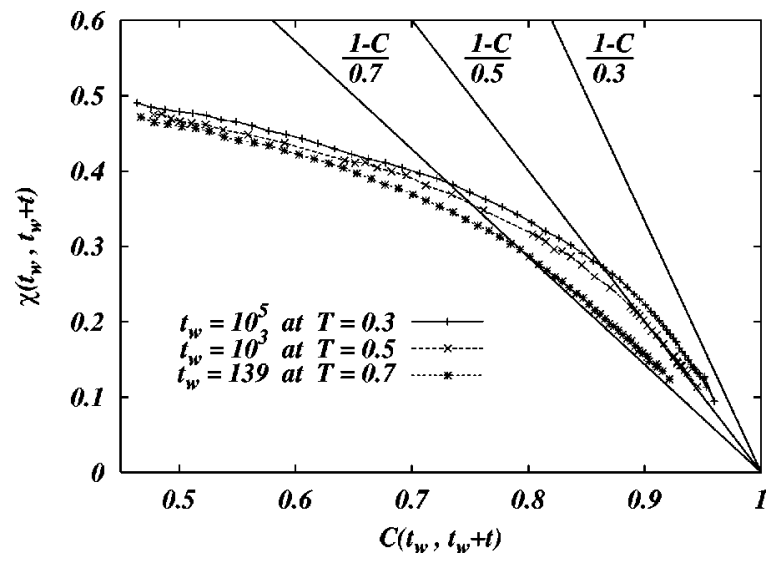

FIG. 5. FDT measurements taken at a temperature $T=0.5$, after the thermalizations shown in the legend. They show that in the quasiequilibrium regime (i.e., in the thermalized regions) the effective temperature of the system is always the maximum between the one used to thermalize the system and the one used to take measurements.

Through the measure of the fluctuation-dissipation ratio we can estimate the effective temperature of the system on those length scales. In Fig. 5 we show the results for the three experiments. The reference data, in the quasiequilibrium regime, perfectly stay on the line $(1-C) / 0.5$ as it should. The other two data sets, because they have been thermalized at temperature $T_{1}$ and then let to evolve at a different temperature $T_{2}=0.5$, fall in between the line $(1-C) / T_{1}$ and $(1$ $-C) / 0.5$, showing that the system temperature is changing from $T_{1}$ to $T_{2}$. However the interesting point to note is that the change is very different in the two cases. For $T_{1}=0.3$ (uppermost curve in Fig. 5) the system responds with an effective temperature very similar to $T_{2}=0.5$, given by the slope in the quasiequilibrium regime. While for $T_{1}=0.7$ (lowest curve in Fig. 5) the effective temperature is perfectly compatible with $T_{1}=0.7$. In general, we would say that if an Edwards-Anderson model is thermalized to a temperature $T_{1}$ and measurements are done at a different temperature $T_{2}$ the response of the system will be dominated by the higher temperature. This is the only effect asymmetric in $\Delta T$ we have found in all the numerical experiments performed with temperature changes.

Recently Bernardi et al. ${ }^{28}$ have proposed the following scaling for the susceptibility $\chi\left(t_{w}, t_{w}+t\right)=\tilde{\chi}\left(\xi\left(t_{w}\right), \xi(t)\right)$, where $\xi(t)$ is the dynamical correlation length defined above. Our data for the susceptibility, which are measured on larger time and temperature scales than Ref. 28 , do not fit that scaling.

\section{MEMORY AND CHAOS IN ac RELAXATIONS}

In this section we present a detailed investigation of memory, rejuvenation and cooling rate effects in the EA model doing ac susceptibility numerical experiments. In what follows we will use indistinctly the words chaotic, rejuvenation or reinitialization to indicate the presence of new relaxational processes which have been driven off- 

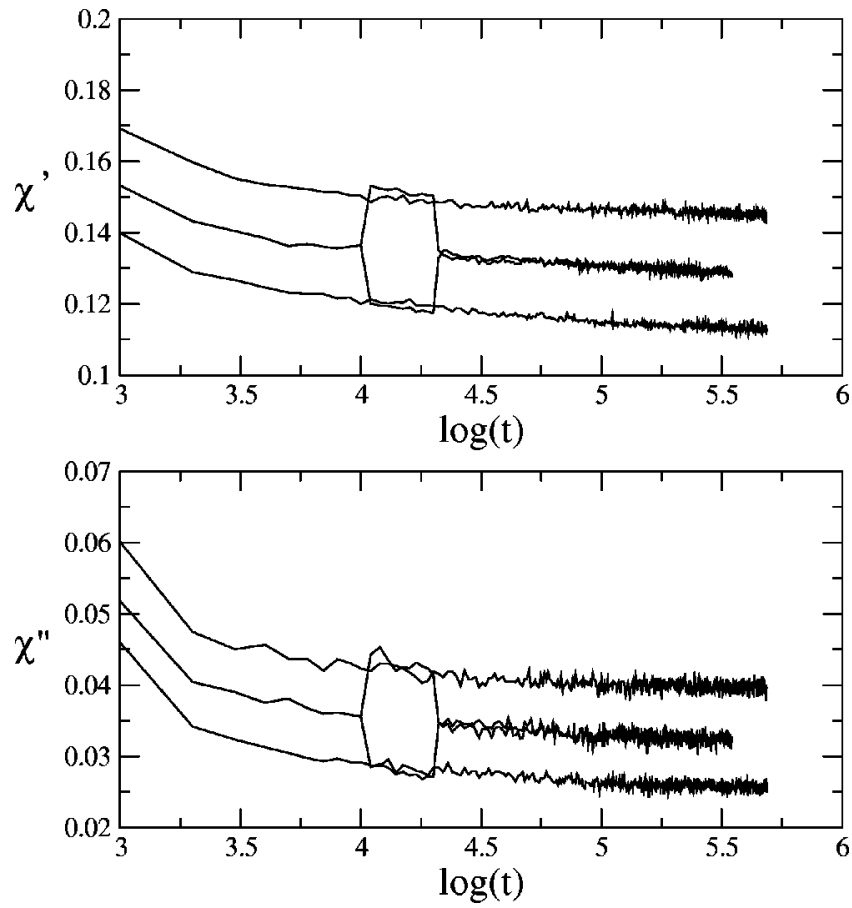

FIG. 6. ac temperature variation experiment. The system is quenched at $T=0.6$ and the ac susceptibility is recorded for a field of period $P=100$ and intensity $h_{0}=0.1$. At time $t_{1}=10000$ the temperature is changed by $\Delta T= \pm 0.1$ and after the same time interval temperature is restored to its original value. For comparison we show the reference relaxation curves at temperatures $T+\Delta T$ $=0.7$ and $T-\Delta T=0.5$

equilibrium when a perturbation is applied. There is a large list of ac experiments in the presence of external perturbations such as temperature field variations or magnetic field variations. $^{3,29}$ The experimental setting is as follows. The system is quenched to a low temperature (ranging between 0.6 and 0.9 times the value of $T_{g}$ ) and the slow decay of the ac susceptibility recorded. After a time $t_{1}$ where $\omega t_{1}$ is such that the ac susceptibility has not totally decayed to its asymptotic value a perturbation is applied. To have an idea, the time $t_{1}$ is such that both $\chi^{\prime}$ and $\chi^{\prime \prime}$ are still between $5 \%$ and $20 \%$, of the whole decay, above their asymptotic large $t$ value. This corresponds to typical values of $\omega t_{1}$ ranging from 100 to 2000. At this time there is a sudden perturbation (for instance, a change in temperature or field). After a time interval $t_{2}$ which is of the same order as $t_{1}$ the perturbation is switched off. In the present study and for sake of simplicity we have taken $t_{2}=t_{1}$. All the times $t$ and frequencies $\omega$ we used in the numerical experiments are such that the scaling $\omega t$ is satisfied. ${ }^{30}$

In the presence of rejuvenation or chaotic effects one generally observes strong reinitialization of the ac susceptibilities corresponding to processes which have been driven offequilibrium as consequence of the perturbation. Having in mind the previous experimental setting we have considered the following different types of perturbations: temperature changes, magnetic field changes and quenched disorder changes.
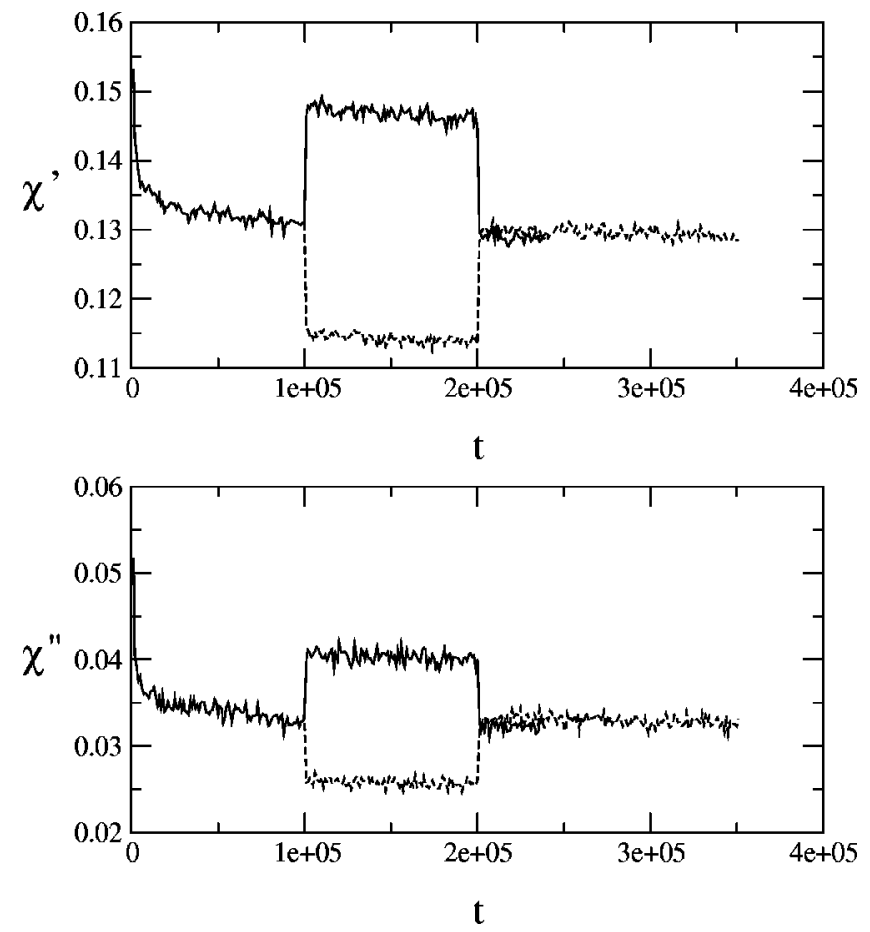

FIG. 7. The same experiment as in Fig. 6 but with $t_{1}$ $=100000$.

\section{A. Temperature changes}

We quench the system to a temperature below $T_{c}$ and apply an ac magnetic field measuring the ac susceptibility. At a given time $t_{1}$ we suddenly change the temperature to $T+\Delta T$ and still measure the ac susceptibility. Then, after a time interval equal to $t_{1}$ we reset again the temperature to its original value $T$. In the presence of chaotic or rejuvenation effects we expect that a sudden change in temperature will reinitialize some relaxational processes. In Fig. 6 we show the results for $L=64$ by measuring relaxation at $T=0.6$ and making two jumps in temperature $\Delta T= \pm 0.1$ at times $t_{1}$ $=10000$ and $t_{1}+t_{2}=2 t_{1}$. The jump in temperature is then applied when a large part of the ac susceptibility is still relaxing like in the experimental setting. Note that for a positive temperature change $\Delta T$ the ac susceptibility stays above the reference curve at the temperature $T+\Delta T$. This means that the effective waiting time after the positive jump is smaller than that of the reference curve at higher temperature. For a negative temperature jump $-\Delta T$ the ac susceptibility stays below the reference curve at temperature $T$ $-\Delta T$. This means that the effective waiting time has now increased and relaxation at $T-\Delta T$ has benefited from relaxation at the higher temperature $T$. The results of Fig. 6 show that the effective time $t_{\text {eff }}$ during the interval of time $t_{2}$ when temperature has been changed is controlled by the same activated processes but with a different activation rate,

$$
t_{\mathrm{eff}}=t_{2}^{(T \pm \Delta T) / T}
$$

implying $t_{\text {eff }}>t_{2}$ if $\Delta T>0$ and vice versa. As comparison we also show a similar plot for $t_{1}=100000$ when nearly all relaxation of ac susceptibility has taken place in Fig. 7. 

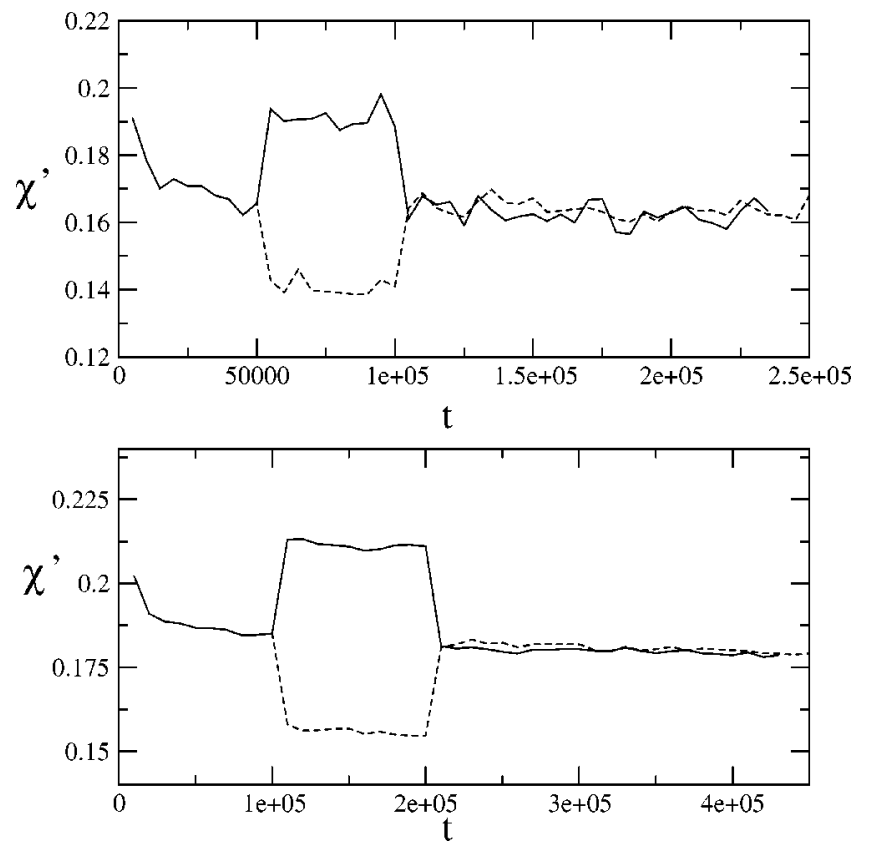

FIG. 8. Plot above: The equivalent experiment of Fig. 6 but with a larger size, a smaller field, and a smaller frequency: $L=100 ; h$ $=0.01 ; P=500$. Plot below: The equivalent experiment of Fig. 6 but with a 10 times smaller frequency $P=1000$. Note that the perturbation in these two experiments and in Fig. 6 are applied when $\omega t=100$.

Again no traces of reinitialization effects in the ac susceptibility are observed. The main feature we can appreciate from the figure is that the positive jump and the time spent at $T$ $+\Delta T$ has increased the effective age of the system respect to the reference temperature $T$ respect to the curve for the negative jump in agreement with Eq. (18). Note in the figure that, after the second jump, the dashed line stays above the continuous line. Note that this temperature dependence of the effective time (18) is the one found in the REM, ${ }^{31,32}$ and here seems to behave quite well.

The picture which emerges from these figures is in agreement with all the results published up to now which point in the direction that there are no chaotic or rejuvenation effects in Edwards-Anderson spin glasses in the presence of temperature changes. One could argue that there are several factors which induce the absence of any trace of rejuvenation. Among them: (1) The intensity of the field which is bigger than in experiments; (2) the smallness of the period of the oscillating field which covers at most nanoseconds when compared to real experiments (of order of seconds); and (3) the size of the system which is large enough. In Fig. 8 we show the same results as Fig. 6 for a field $h_{0}=0.01$, a larger size $L=100$ and a larger period $P=500$ such that $\omega t$ takes the same value, so we are in the same time scale according to the results of Ref. 30. Due to the smallness of the probing field the signal is now much more noisy so we show the in-phase susceptibility. The smallness of the absolute magnitude of the time scales involved in numerical experiments [reason (2) above] is usually advocated as the main source of discrepancy between numerics and experiments. The lower plot in Fig. 8 shows $\chi^{\prime}$ for the same size and field as Fig. 6 but with a frequency 10 times smaller. The conclusions are exactly the same: cooling rate effects are important and no trace of reinitialization after the temperature variation is observed.

\section{B. Field and coupling distribution variations}

To make evident how much this absence of chaos or rejuvenation is indeed an intrinsic effect to temperature changes we have done the same experiment with a different type of perturbation. Instead of changing the temperature, we have applied a perturbation which is well known to be chaotic from equilibrium studies. Examples of such perturbations are (1) a change in the uniform magnetic field ${ }^{33,34}$ and (2) a change in the couplings distribution. ${ }^{35}$

Concerning the first type of variation there have been several experiments which reveal how reinitialization occurs under a dc magnetic field change. ${ }^{29}$ The experimental setting is the same as that shown previously but now the perturbation is to apply a dc magnetic field after $t_{1}$. So the system is quenched at zero dc field and at time $t_{1}$ the dc field is switched on. After a time interval $t_{2}=t_{1}$ the dc field is set to zero again. In laboratory experiments ${ }^{29}$ the intensity of the applied dc field must be larger than the amplitude of the ac field for the ac field to probe the response of the system after the dc perturbation. The intensity of the probing ac field is typically smaller than one Oersted and the intensity of the ac field much higher (between 5 and 10 Oersteds). So, typically the intensity of the dc field is 10 times or even more larger than the probing ac field. Nevertheless, in the numerical experiments we have a problem. The intensity of the ac field cannot be arbitrarily small, otherwise we have a too small and noisy signal. Consequently, if the perturbing dc field is chosen 10 times larger than the ac field then the resultant field $(\mathrm{ac}+\mathrm{dc})$ will be very large and drive the system out of the linear response regime. Moreover, nonlinear effects will be much enhanced because of the nonlinear coupling between the perturbation (the dc field) and the probing field (the ac field). A way to avoid this is to apply a perturbing dc field which does not couple with the probing field, for instance, a staggered dc field. Now the perturbation is given by a new term $\delta \mathcal{H}$ in the Hamiltonian

$$
\delta \mathcal{H}=-h_{\mathrm{dc}} \sum_{i=1}^{V} \epsilon_{i} \sigma_{i},
$$

where $\epsilon_{i}$ are quenched random variables which may take the values \pm 1 . Another equivalent procedure would be to apply a uniform dc field as perturbation and measuring the ac susceptibility corresponding to the response to a staggered probing ac field. The results are shown in Fig. 9. Note that strong reinitialization is seen after perturbing the system in agreement with the known result that finite-dimensional spin glasses are chaotic against magnetic field changes. ${ }^{34}$

A similar result is found by considering the other type of perturbation. In that case we measure the ac susceptibility after quenching at temperature $T$. At $t_{1}$ we take a percentage $r(0<r<1)$ of the couplings and reverse its sign $J_{i j}$ $=-J_{i j}$. After a new interval $t_{1}$ we reput the original cou- 

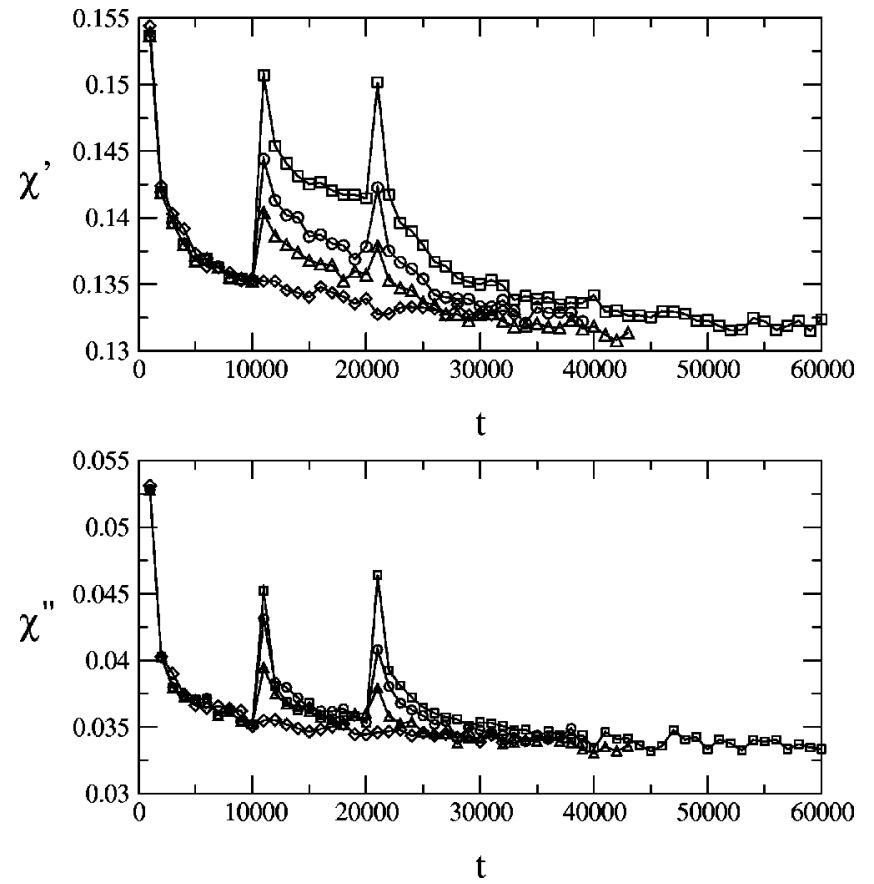

FIG. 9. dc field variation experiment. The system $L=100$ is quenched at $T=0.6$ and the ac susceptibility is recorded for a probing field of period $P=100$ and intensity $h_{0}=0.1$. At time $t_{1}$ $=10000$, a dc field is applied for a time interval $t_{2}=t_{1}$. After $t_{1}$ $+t_{2}$ the field is switched off. The intensities of the dc fields are $h_{\mathrm{dc}}=0.4$ (triangles), 0.6 (circles), and 1.0 (squares). The diamonds correspond to the reference curve without perturbation.

plings again. The results are shown in Fig. 10 for $t_{1}$ $=10000, \omega=0.01$, and $r=0.05,0.1$ (corresponding to $5 \%$ and $10 \%$ of changes in the couplings respectively). Note the presence of strong and clear reinitialization effects in agreement with the fact that such a perturbation is chaotic.

We may conclude this section saying that while there is clear trace of chaotic behavior in the presence of field or couplings changes, there is absolutely no trace of reinitialization effects below the spin-glass transition in the case of temperature change experiments. This may be due to the presence of cooling rate effects in three dimensional Ising spin glasses stronger than those measured in laboratory experiments.

\section{OUTLOOK AND DISCUSSION}

Two years ago some experimentalists from the Saclay and the Uppsala groups measured on a spin glass sample very strong memory and chaos effects. ${ }^{6}$ Their results are really impressive and show unambiguously how important are these effects in real spin glasses. By investigating the Edwards-Anderson model in three dimensions we have tried to reproduce numerically their findings, but we have obtained results pointing in the opposite direction. Temperature variation experiments in spin glasses are nowadays one of the most puzzling results in the field. It is unclear which is the final theory which may naturally account for these results. It is not easy to explain, from the point of view of the droplet model, ${ }^{36}$ how reorganization of domains can account
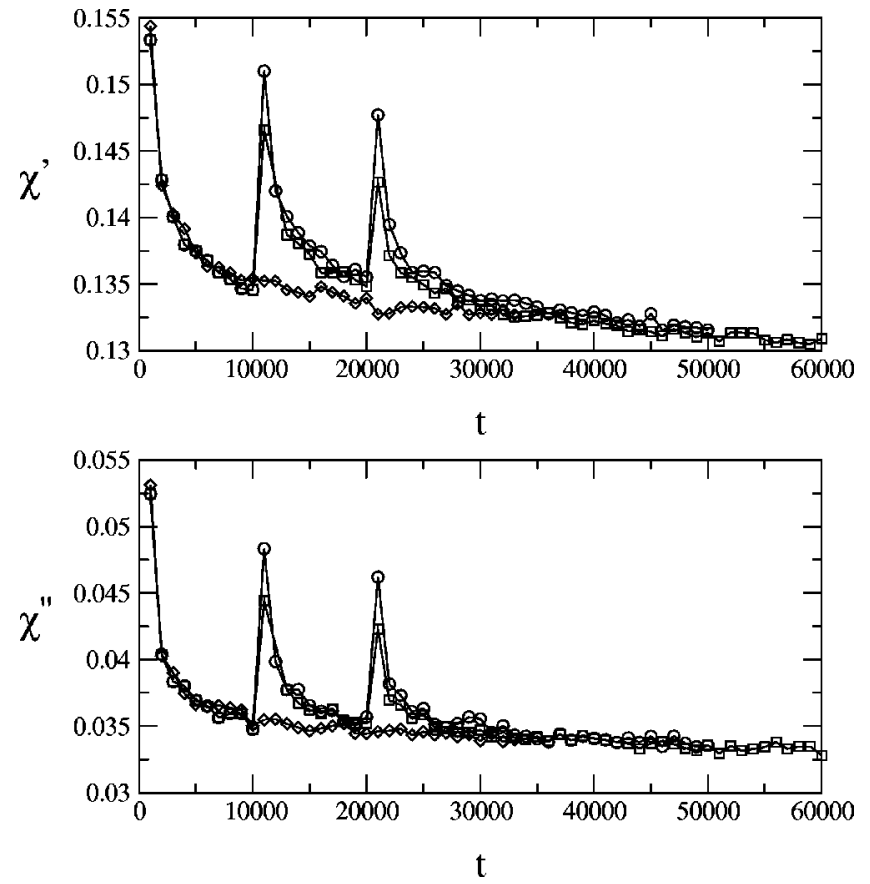

FIG. 10. Couplings variation experiment. The system $L=100$ is quenched at $T=0.6$ and the ac susceptibility is recorded for a probing field of period $P=100$ and intensity $h_{0}=0.1$. At time $t_{1}$ $=10000$, a percentage $r$ of the couplings change sign and the ac susceptibility is recorded for a time interval $t_{2}=t_{1}$. After $t_{1}+t_{2}$ the couplings again take their original values. The intensities of the perturbation are $r=0.05$ (squares) and $r=0.1$ (circles). The diamonds correspond to the reference curve without perturbation.

for the memory effects observed in the experiments. ${ }^{6,7,37} \mathrm{Al}-$ ready in equilibrium theory, the question whether the spinglass phase is chaotic against temperature changes is far from being settled. Extensive numerical work does not show any clear evidence of chaos for temperature changes. ${ }^{34,38-40}$

Regarding off-equilibrium dynamics the situation is similar. Very precise numerical simulations by Rieger et al. ${ }^{12}$ show that correlations between equilibrium configurations at different temperatures are big and the corresponding overlap length grows algebraically in time without any tendency to saturation within the simulated range of times. The same conclusions hold for TRM numerical experiments with temperature change protocols. ${ }^{13}$ Very recently, Komori et al. ${ }^{14}$ have presented a detailed study of the two-time correlations in the presence of temperature change variations. These should be essentially equivalent to the present results because ac experiments probe the quasistationary aging regime where fluctuation-dissipation makes responses and correlations equivalent. Their conclusion is like ours: no rejuvenation effects are found and cooling rate effects are very strong. The only claimed evidence for rejuvenation and memory effects is Fig. 7 in that reference which is unconclusive as we have explained in Sec. IV. Contrarily, the results for dc field variation experiments resemble quite much those of experiments and, together with coupling distribution variations, show that chaotic effects manifest as reinitialization effects in the ac susceptibility.

What is the origin of this discrepancy between experi- 
ments and simulations? We indicate three possible reasons: (1) The size of the system is too small. (2) The intensity of the probing field is too large. (3) The absolute magnitude of the time scales in numerical experiments are too small.

As we showed in Fig. 8 improvement in these limitations (larger size, smaller frequency, smaller ac field) does not seem to alter the final conclusions. Unfortunately we cannot still definitely conclude anything because probably our improvements (within the available computer capabilities) are too modest. Let us briefly comment about these three possibilities. Probably the finiteness of the size is the less important. A larger size diminishes statistical fluctuations but has no strong effect on the growing domains because they are known to be very small. ${ }^{41,12,21,42}$ Regarding the smallness of the field we have shown in Fig. 8 that a field 10 times smaller does not change the conclusions and for this smaller value of the probing ac field we are much closer in magnitude to the experimental setup. Moreover, field-cooling and zero-field cooling experiments, using the same values for the external dc field, show that the system is in the linear response regime. ${ }^{21,43}$ There are no deep reasons why things should drastically change for an ac probing field 100 times smaller to the one used in the present simulations. At most the intensity of the field can give non negligible corrections to the usual $t / t_{w}$ scaling ${ }^{44}$ as shown in Ref. 30 .

The smallness of time scales involved (i.e., the fact that ac frequencies are too large and the waiting times too small) is the most serious reason and could definitively be the origin of the discrepancy. Short-time scales obviously imply short length scales of size $\xi(P)$ or $\xi\left(t_{w}\right)$ depending on the kind of experiment. If temperature changes would have no effect on small length scales but only on very large scales then chaotic effects could not be seen in standard numerical simulations. This statement corresponds to saying that the overlap length $L(\Delta T)$ for a typical temperature change $\Delta T$ is much larger than any probed domain length in the numerical experiment, but smaller than those probed in the laboratory (where the chaos is clear). Let us try to quantify more this statement. In laboratory experiments ${ }^{45}$ typically length scales of the order of $\xi \sim 10^{2}$ can be reached, while in numerical simulations we are restricted to length scales between 3 and 5. ${ }^{12,27}$ This discrepancy may or may not be a deep trouble depending on the value of the chaos exponent. Unfortunately there is not a numerical estimate for the chaos exponent (because chaos in temperature has never been observed) and we only have an estimate from domain-wall scaling arguments ${ }^{46}$ which give $L(\Delta T)=(\Delta T)^{-\zeta}$ with $\zeta=d_{s} / 2-\theta$ where $d_{s}$ is the fractal surface of droplets and $\theta$ is the thermal exponent. For $d$ $=3$, the value of $\zeta$ must be larger than 1 and a reasonable value seems to be $\zeta \simeq 1.5,{ }^{47}$ so chaos in temperature should not be too small after all. Note also that for a dc field change the chaos exponent is even smaller, the overlap length being given by $L(h) \sim h^{-2 / 3} \cdot{ }^{34}$ So, for similar values for the prefactor, one would expect stronger chaos in temperature than in a dc field. On top of that, the analysis by Bray and Moore $^{46}$ for the value of the chaos exponent $\zeta$, shows that is consequence of the balance between a contribution coming from the surface of the droplets $L^{d_{s} / 2}$ and a contribution from the activation energy necessary to revert a droplet $L^{\theta}$. The surface contribution comes from the inhomogeneities in the couplings on the surface and, actually, the same type of analysis should fully carry through when analyzing chaos for coupling perturbations. But in this last case we found strong reinitialization effects (see Fig. 10) which are absent when changing the temperature. How this difference which we observe can be explained in the framework of the droplet model remains mysterious unless one advocates different prefactors for both overlap lengths (much bigger for temperature changes than for couplings changes) or that something special occurs in three dimensions. ${ }^{48}$ With the present estimations for the $\zeta$ exponent and if the prefactor for the overlap length is very large, chaotic effects, which experimentally appear on temperature changes such that $\Delta T$ $\simeq 0.1 T_{c}$, may need in present simulations temperature changes of the same order of the absolute temperature. If this is the case then we have to wait for the next computer generation or to study the ea model in a situation such that larger length scales could be reached (e.g., in $4 \mathrm{D}$ or in $3 \mathrm{D}$ with next-nearest neighbors interactions).

On the other hand, our results imply that if the laboratory experiments were done at frequencies of $10^{9} \mathrm{Hertz}$ (instead of the typical $1 \mathrm{~Hz}$ measurements) then cooling rate effects would be restored and rejuvenation or chaotic effects disappear. Unfortunately, there are no experiments in the range $10-10^{9}$ Hertz. Note also that, according to the general $\omega t$ scaling, for these frequencies one should do measurements at very short times such that $\omega t$ is not much larger than $10^{4}$ and the ac susceptibility has not completely relaxed. Still, the results of Ref. 30, the experimental results of Ref. 3 and all the numerical published data up to now show that the scaling $\omega t$ (the equivalent of the scaling $t / t_{w}$ in two-time experiments) works reasonably well (with some slight deviations). If the scaling $t / t_{w}$ means something (as most of the present theoretical work suggests) then it is difficult to understand why no trace of reinitialization effects is observed in the smallest coarsening domains when the temperature is changed. Actually these reinitialization effects are found for dc magnetic field and coupling distribution changes. If some dynamical effects are completely absent for the small coarsening sizes this means that no numerical simulation in the last ten years has actually reached the asymptotic regime where connection to real experiments is possible and we are certainly missing something. ${ }^{37}$

Concerning theory, it is difficult to give a complete description on these effects in terms of compact excitations as proposed in the droplet model. ${ }^{36}$ Usually the asymmetric response of the spin glass against the sign of the temperature perturbation is explained in terms of an asymmetric overlap length $L(\Delta T)$. The problem is that in simulations $L(\Delta T)$ is apparently extremely large when compared to experiments and also symmetric, a question which is difficult to explain again if one does not appeal to the smallness of the time scales involved. If the necessary time scales to see the asymmetric effects in $L(\Delta T)$ exceed the experimental time scales $^{49}$ then it is unclear how to reconcile any two among the three: theory, experiments and simulations.

The explanation of memory and chaotic effects was originally explained in terms of a hierarchical picture. ${ }^{3}$ Actually, 
these effects are explained and reproducible in the GREM, ${ }^{50,51}$ a model with several critical temperatures. The same phenomena is absent in the random-energy model ${ }^{31,32}$ with a single critical temperature. Unfortunately, the GREM is a model without microscopic and spatial description so the connection with real spin glasses remains speculative. As we said previously the results we find here, and in particular the cooling rate effects, are very similar to those of the random energy model..$^{32}$ It is well known that the spin-glass phase in this model can be described in terms of a one-step solution. And what we see in our simulations is what is expected for a mean-field model with a one-step of replica symmetry breaking solution. ${ }^{52}$ This solution is known to describe the physics behind structural glasses. ${ }^{5}$ Actually temperature variation experiments on structural glasses ${ }^{53-55}$ resemble our simulation results much more than what experiments on spin glasses do. Again one could claim that the one-step character of the effects we observe in our simulations are due to the smallness of time scales and if one increases the time scales (let us say by 6 orders of magnitude) much different results will come out.

But there could be another and more natural explanation. Is the Edwards-Anderson model really a good spin glass? Is it possible that the Edwards-Anderson model has strong cooling rate effects and no rejuvenation effects at all? If rejuvenation or chaotic effects are present in the dynamics for a given perturbation this could be related to the fact that equilibrium properties are chaotic when such a perturbation is switched on. Analytical calculations in the mean-field version of the Edwards-Anderson model (the SherringtonKirkpatrick model) confirming chaoticity for temperature changes are still inconclusive. ${ }^{40,56}$ A completely different scenario holds for field and coupling perturbations in agreement with the present simulations. Let us note also that real spin glasses are site disordered systems, a type of disorder not included in the Edwards-Anderson model. Recent experiments on the Kagome antiferromagnet lattice ${ }^{57}$ reveal that there are not strong reinitialization effects after changing the temperature. Because that system does not include disorder at all one may wonder whether the influence of site disorder can be important. Site-disordered spin glasses were not much considered in the past (for some works and references, see Ref. 58) because they were thought to be less relevant to experiments than bond-disordered models but probably this is not true and a site disorder effect should be taken into account to explain experiments. To finish this collection of possible ways out to this puzzle let us point out also the possible role of the continuous character of the spins in a real spin glass as well as the effect of chirality. ${ }^{59}$ This last effect and its importance in the description of the dynamics of spin glasses will surely see further developments in the forthcoming years.

It is clear that we are facing a very difficult problem. If the smallness of time scales of the simulation is the final explanation for everything then a sensible theory for spin glasses must explain why temperature changes are fundamentally so peculiar when compared to other type of perturbations, i.e., why the prefactor for the overlap length $L(\Delta T)$ is so large. In this respect, experiments at much larger frequencies are necessary. At least, to see if cooling rate effects gradually change and reinitialization effects, in the presence of temperature variations, do systematically weaken. On the other hand, if the Edwards-Anderson model fails to explain a crucial result found in experiments then we must discover what ingredient is lacking in the original model and what are the consequences for our present knowledge of spin glass theory. In this direction, finding a microscopic model with spatial structure which presents a spin-glass phase transition and unambiguously shows memory, chaotic as well as absence of cooling rate effects would be welcome.

\section{ACKNOWLEDGMENTS}

We are grateful to J.P. Bouchaud, M. Mezard, G. Parisi, and $\mathrm{E}$. Vincent for useful discussions and $\mathrm{H}$. Rieger and $\mathrm{H}$. Takayama for useful correspondence. M.P. and F.R. acknowledge financial support from a French-Spanish collaboration (Picasso program and Acciones Integradas Ref. HF1998-0097).
*Electronic address: picco@1pthe.jussieu.fr

†Electronic address: riccife@ictp.trieste.it

Electronic address: ritort@ffn.ub.es

${ }^{1}$ L.C.E. Struik, Physical Aging in Amorphous Polymers and Other Materials (Elsevier Scientific, Amsterdam, 1978).

${ }^{2}$ L. Lundgren, P. Svedlindh, P. Nordblad, and O. Beckman, Phys. Rev. Lett. 51, 911 (1983).

${ }^{3}$ E. Vincent, J. Hamman, M. Ocio, J.P. Bouchaud, and L.F. Cugliandolo, in Complex Behavior in Glassy Systems, Proceedings of the XIV Sitges Conference, 1996, edited by M. Rubí and C. Perez-Vicente (Springer-Verlag, Berlin, 1997).

${ }^{4}$ P. Nordblad and P. Svedlindh, in Spin Glasses and Random Fields, Series on Directions in Condensed Matter Physics Vol. 12, edited by A.P. Young (World Scientific, Singapore, 1998).

${ }^{5}$ J.P. Bouchaud, L.F. Cugliandolo, J. Kurchan, and M. Mezard, in Spin Glasses and Random Fields (Ref. 4).

${ }^{6}$ K. Jonason, E. Vincent, J. Hamman, J.P. Bouchaud, and P. Nordblad, Phys. Rev. Lett. 81, 3243 (1998).
${ }^{7}$ J. Hammann, E. Vincent, V. Dupuis, M. Alba, M. Ocio, and J.P. Bouchaud, cond-mat/9911269 (unpublished).

${ }^{8}$ T. Jonsson, K. Jonason, P. Jönsson, and P. Nordblad, Phys. Rev. B 59, 8770 (1999); T. Jonsson, K. Jonason, and P. Nordblad, ibid. 59, 9402 (1999).

${ }^{9}$ Results in a ferromagnetic fine particle system are shown in $\mathrm{H}$. Mamiya, I. Nakatani, and T. Furubayashi, Phys. Rev. Lett. 82, 4332 (1999).

${ }^{10}$ E. Marinari, G. Parisi, and J.J. Ruiz-Lorenzo, in Spin Glasses and Random Fields (Ref. 4).

${ }^{11}$ The most recent evidence for a finite-temperature phase transition in 3D spin glasses is for the $\pm J$ model: N. Kawashima and A.P. Young, Phys. Rev. B 53, R484 (1996); M. Palassini and S. Caracciolo, Phys. Rev. Lett. 82, 5128 (1999); A. Hartmann, Phys. Rev. E 59, 84 (1999); H.G. Ballesteros et al., Phys. Rev. B 62, 14237 (2000).

${ }^{12}$ J. Kisker, L. Santen, M. Schreckenberg, and H. Rieger, Phys. Rev. B 53, 6418 (1996). 
${ }^{13}$ H. Rieger, J. Phys. I 4, 883 (1994); Physica A 224, 267 (1996).

${ }^{14}$ T. Komori, H. Yoshino, and H. Takayama, cond-mat/0001395 (unpublished).

${ }^{15}$ J.O. Andersson, T. Jonsson, and J. Mattson, Phys. Rev. B 54, 9912 (1996).

${ }^{16}$ S.F. Edwards and P.W. Anderson, J. Phys. F: Met. Phys. 5, 965 (1975).

${ }^{17}$ K. Binder and A.P. Young, Rev. Mod. Phys. 58, 801 (1986); K. Fischer and J. Hertz, Spin Glasses (Cambridge University Press, Cambridge, England, 1991).

${ }^{18}$ The Ising character of Ising spin glasses is supported by the fact that Heisenberg spin glasses do not have a finite temperature phase transition.

${ }^{19}$ C. Battista et al., Int. J. High Speed Comput. 5, 637 (1993).

${ }^{20}$ S. Franz and H. Rieger, J. Stat. Phys. 79, 749 (1995).

${ }^{21}$ E. Marinari, G. Parisi, F. Ricci-Tersenghi, and J.J. Ruiz-Lorenzo, J. Phys. A 31, 2611 (1998).

${ }^{22} \mathrm{We}$ indistinctly refer to both the in-phase and out-of-phase components.

${ }^{23}$ J. Hammann, M. Ocio, and E. Vincent, in Relaxation of Complex Systems and Related Topics, edited by I. Campbell and C. Giovanella (Plenum, New York, 1990).

${ }^{24} \mathrm{E}$. Vincent (private communication).

${ }^{25} \mathrm{H}$. Takayama (private communication).

${ }^{26}$ G. Parisi, F. Ricci-Tersenghi, and J.J. Ruiz-Lorenzo, J. Phys. A 29, 7943 (1996).

${ }^{27}$ E. Marinari, G. Parisi, F. Ricci-Tersenghi, and J.J. Ruiz-Lorenzo, J. Phys. A 33, 2373 (2000).

${ }^{28}$ L.W. Bernardi, H. Yoshino, K. Hukushima, H. Takayama, A. Tobo, and A. Ito, Phys. Rev. Lett. 86, 720 (2001).

${ }^{29}$ E. Vincent, J.P. Bouchaud, J. Hammann, and F. Lefloch, Philos. Mag. B 71, 489 (1995); E. Vincent, J.P. Bouchaud, D.S. Dean, and J. Hammann, Phys. Rev. B 52, 1050 (1995).

${ }^{30}$ M. Picco, F. Ricci-Tersenghi, and F. Ritort, cond-mat/0005541, Eur. Phys. J B (to be published).

${ }^{31}$ B. Derrida, Phys. Rev. B 24, 2613 (1981).

${ }^{32}$ M. Sasaki and K. Nemoto, cond-mat/0002021 (unpublished).

${ }^{33}$ I. Kondor, J. Phys. A 22, L163 (1989).

${ }^{34}$ F. Ritort, Phys. Rev. B 50, 6844 (1994).

${ }^{35}$ V. Azcoiti, E. Follana, and F. Ritort, J. Phys. A 28, 3863 (1995).

${ }^{36}$ W.L. McMillan, J. Phys. C 17, 3179 (1984); A.J. Bray and M.A. Moore, ibid. 18, L699 (1985); D.S. Fisher and D.A. Huse, Phys. Rev. B 38, 373 (1988); 38, 386 (1988).

${ }^{37}$ For a recent discussion, see J.P. Bouchaud, in Soft and Fragile Matter: Nonequilibrium Dynamics, Metastability and Flow, ed- ited by M. E. Cates and M. R. Evans (IOP Publishing, Philadelphia, 2000), pp. 285-304.

${ }^{38}$ I. Kondor and A. Vegsö, J. Phys. A 26, L641 (1993); S. Franz and M. Nifle, ibid. 28, 2499 (1995).

${ }^{39}$ M. Nifle and A.P. Young, J. Phys. A 30, 5311 (1997); M. Nifle, Phys. Rev. B 57, 492 (1998).

${ }^{40}$ For very recent results, see E. Marinari and A. Billoire, cond-mat/9910352 (unpublished).

${ }^{41}$ D. Huse, Phys. Rev. B 43, 8673 (1991).

${ }^{42}$ T. Komori, H. Yoshino, and H. Takayama, J. Phys. Soc. Jpn. 68, 3387 (1999).

${ }^{43}$ G. Parisi, F. Ricci-Tersenghi, and J.J. Ruiz-Lorenzo, Eur. Phys. J. B 11, 317 (1999).

${ }^{44}$ G. Parisi, Philos. Mag. B 71, 471 (1995).

${ }^{45}$ Y.G. Joh, R. Orbach, G.G. Wood, J. Hammann, and E. Vincent, Phys. Rev. Lett. 82, 438 (1999).

${ }^{46}$ A.J. Bray and M.A. Moore, Phys. Rev. Lett. 58, 57 (1987).

${ }^{47}$ M. Palassini and A.P. Young, Phys. Rev. Lett. 85, 3017 (2000); F. Krzakala and O.C. Martin, ibid. 85, 3013 (2000).

${ }^{48}$ M. Nifle and H. Hilhorst, Phys. Rev. Lett. 68, 2992 (1992); Physica A 193, 48 (1993).

${ }^{49}$ A scenario where critical effects influence strongly the spin glass phase close to $T_{g}$ leading to extremely high crossover time scales has been proposed by H. Bokil, B. Drossel, and M.A. Moore, cond-mat/0002130 (unpublished).

${ }^{50}$ B. Derrida, J. Phys. (France) Lett. 46, L401 (1985).

${ }^{51}$ M. Sasaki and K. Nemoto, J. Phys. Soc. Jpn. 69, 2283 (2000).

${ }^{52}$ L.F. Cugliandolo and J. Kurchan, Phys. Rev. B 60, 922 (1999).

${ }^{53}$ R.L. Leheny and S.R. Nagel, Phys. Rev. B 57, 5154 (1998).

${ }^{54}$ L. Bellon, S. Ciliberto, and C. Laroche, cond-mat/9905160, Europhys. Lett. (to be published); cond-mat/9906162, Europhys. Lett. (to be published).

${ }^{55}$ F. Alberici, P. Doussineau, and A. Levelut, Europhys. Lett. 39, 329 (1997); F. Alberici, J.P. Bouchaud, L.F. Cugliandolo, P. Doussineau, and A. Levelut, Phys. Rev. Lett. 81, 4987 (1998).

${ }^{56}$ T. Rizzo, cond-mat/0103375 (unpublished).

${ }^{57}$ A.S. Willis, V. Dupuis, E. Vincent, J. Hamman, and R. Calemczuk, cond-mat/0001344 (unpublished).

${ }^{58}$ Th.M. Nieuwenhuizen, Europhys. Lett. 24, 797 (1993); D.S. Dean and D. Lancaster, Phys. Rev. Lett. 77, 3037 (1996); Th.M. Nieuwenhuizen and C.N.A. Van Duin, Eur. Phys. J. B. 7, 191 (1999); E. Marinari, V. Martín-Mayor, and A. Pagnani, Phys. Rev. B 62, 4999 (2000).

${ }^{59}$ H. Kawamura, Phys. Rev. Lett. 68, 3785 (1992); Int. J. Mod. Phys. A 7, 345 (1996); H. Hukushima and K. Kawamura, Phys. Rev. E 61, R1008 (2000). 